\title{
Very long-term follow-up after in-tunnel patent foramen ovale closure with FlatStent EF: still an attractive option?
}

\author{
Eustaquio M. Onorato ${ }^{1}$, Vittorio Ambrosini ${ }^{2}$, Francesco Casilli ${ }^{3}$, Antonio L. Bartorelli ${ }^{1,4}$ \\ ${ }^{1}$ Centro Cardiologico Monzino, IRCCS, University of Milan, Milan, Italy \\ ${ }^{2}$ Reparto di Cardiologia, Ospedale San Giuseppe Moscati, Avellino, Italy \\ ${ }^{3}$ Centro di Cardiologia Interventistica, Istituto Clinico Sant'Ambrogio, Milan, Italy \\ "Department of Biomedical and Clinical Sciences, "Luigi Sacco", University of Milan, Milan, Italy
}

Adv Interv Cardiol 2021; 17, 3 (65): 319-321

DOI: https://doi.org/10.5114/aic.2021.109155

We present a case of a 38-year-old woman, aura migraineur, with a past medical history of Hashimoto's thyroiditis and elevated homocysteine level of $75 \mu \mathrm{mol} / \mathrm{l}$ due to homozygous C677T MTHFR gene mutation on folic acid supplementation. In December 2009 a right-sided hemiparesis on awakening occurred and brain magnetic resonance imaging confirmed left thalamic infarct. Continuous ECG monitoring ruled out atrial fibrillation. A Doppler study of lower limbs revealed no overt thrombosis. Extracranial duplex ultrasound examination was normal and carotid dissection was excluded. Two-dimensional (2D) transthoracic/transesophageal echocardiography (TTE/ TEE) color Doppler showed a right-to-left shunt (RLS) via a long tunnel-type patent foramen ovale (PFO), with an overlap between the primum and secundum septum of $13 \mathrm{~mm}$ along with a prominent Eustachian valve. A contrast-enhanced transcranial Doppler (c-TCD) showed RLS with 10 bubbles in basal conditions and 70 bubbles with a shower pattern following Valsalva strain. Following heart-brain team discussion, transcatheter PFO closure was recommended and the patient was transferred to our institution in January 2010. After written informed consent, she underwent percutaneous PFO closure under mild sedation, with fluoroscopic and rotational intracardiac echocardiography (rICE) guidance using a 9F-9MHz Ultra ICE catheter-based ultra-sound probe (Boston Scientific Corporation, USA), as previously described [1]. Aiming to provide a minimalistic solution to promote closure from within the PFO tunnel, a 19-mm Coherex FlatStent (FS, Coherex Medical, Salt Lake City, USA) [2] was selected based on a stretched tunnel diameter of $>12 \mathrm{~mm}$. This closure system consisted of a flat self-expanding nitinol wire frame covered with polyurethane foam and stabilized within the PFO tunnel by a pair of right and left atri- al anchors (Figure 1). The FS was successfully deployed (Figure 2) and intra-procedural c-TCD and saline contrast infusion-rICE confirmed the immediate and complete abolition of the shunt (Figure 3). The patient was discharged home the next day in good clinical conditions on dual antiplatelet therapy for 1 month. 2D TTE color Doppler showed no residual shunt and a well-aligned in-tunnel device with a minimal exposed surface area and no distortion of the native anatomy, preserving easy future access to the left atrium. The patient recovered fully after her stroke and underwent regular follow-up visits at 1, 6 and 12 months post-procedurally and yearly thereafter. No long-term device-related complications such as new onset atrial arrhythmias, thromboembolism, embolization or erosion of the atrial or aortic wall were documented. Her latest 2D TTE color Doppler performed 11 years after the procedure showed no residual shunt and the FS totally embedded into the septum, being impossible to discern the implanted device from native tissue (Figure 4).

Long-tunnel PFO anatomies still constitute a challenge. In selected patients the in-tunnel approach to PFO closure turned out to be safe, effective and comparable to widely used conventional double-disc devices in terms of complication and closure rates [3-5]. In contrast to bioabsorbable or suture-mediated closure devices, FS provides a more physiologic closure mechanism such as polymer substrate to promote cellular integration within the tunnel. Although FS has been pulled from the market, this innovative closure system may still be largely suitable not only in patients with a long and non-compliant tunnel, thick septum secundum, or large Eustachian valve, but also in tunnel-type PFO. Further technological advances (smaller delivery systems and polyurethane foam replacement with other forms of biological materi-

Corresponding author:

Dr Eustaquio Maria Onorato, Centro Cardiologico Monzino, IRCCS, Italy, phone: +39 348 6939883, e-mail: eustaquio.onorato@gmail.com Received: 12.05 .2021 , accepted: 28.06.2021. 


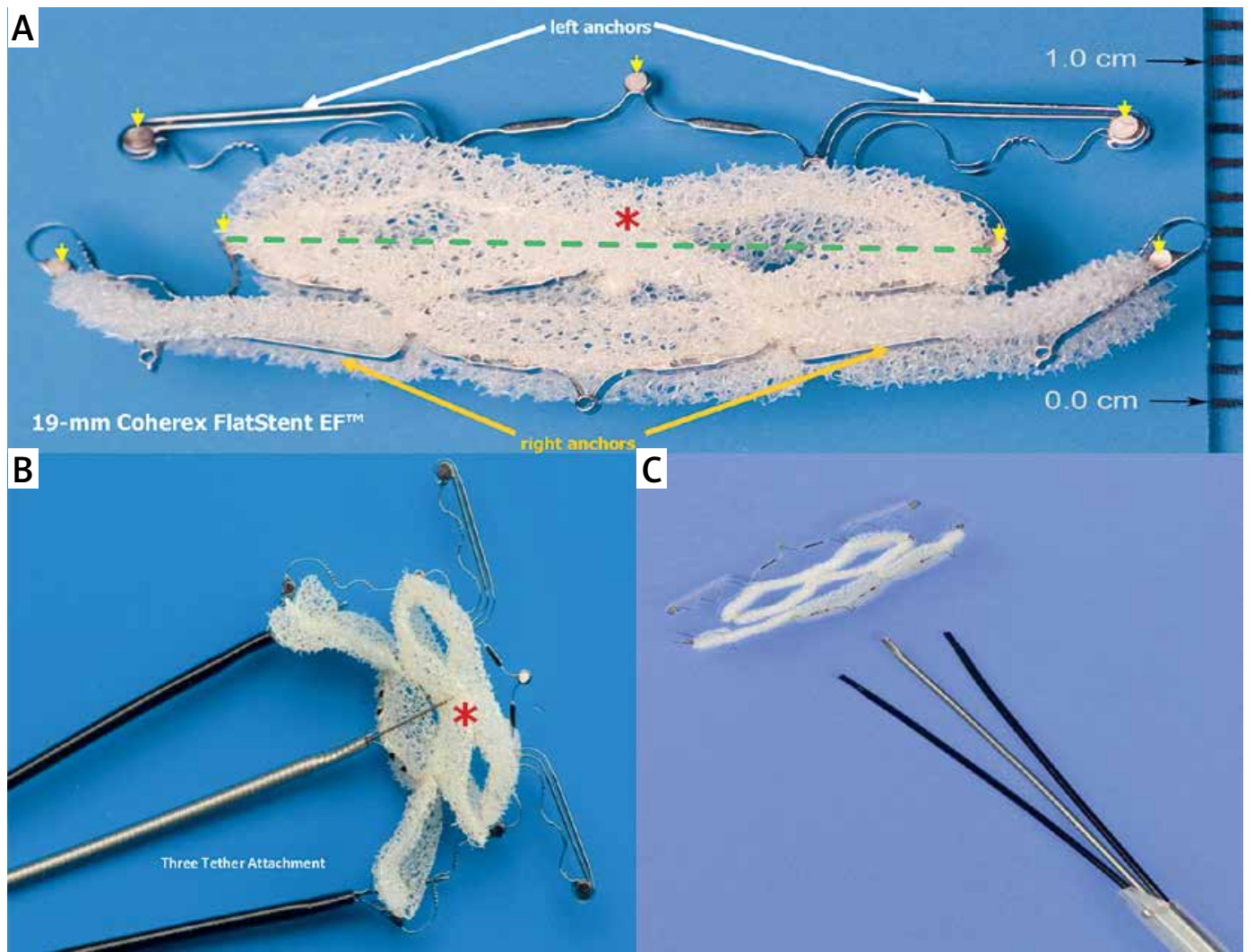

Figure 1. The Coherex FlatStent EF (FS) PFO Closure System. A - The implant is a 0.51-mm thick super-elastic flat nitinol component available in two sizes, 13- and 19-mm, corresponding to the width of the center device portion intended to lie within the PFO tunnel (green dotted line); it has enhanced polyurethane foam (red asterisk) attached around the central cells of the structure; the device has two distal (white arrows) and two proximal (orange arrows) micro-tined anchors that extend out from the PFO tunnel and attach to the walls of the left and right atrium; seven radiopaque markers (yellow small arrows) allow for visualization of the anchors during device placement; $\mathbf{B}$ - the FS pre-attached to a monorail delivery system by three tethers; C - FS released via a simple button mechanism on the delivery system handle
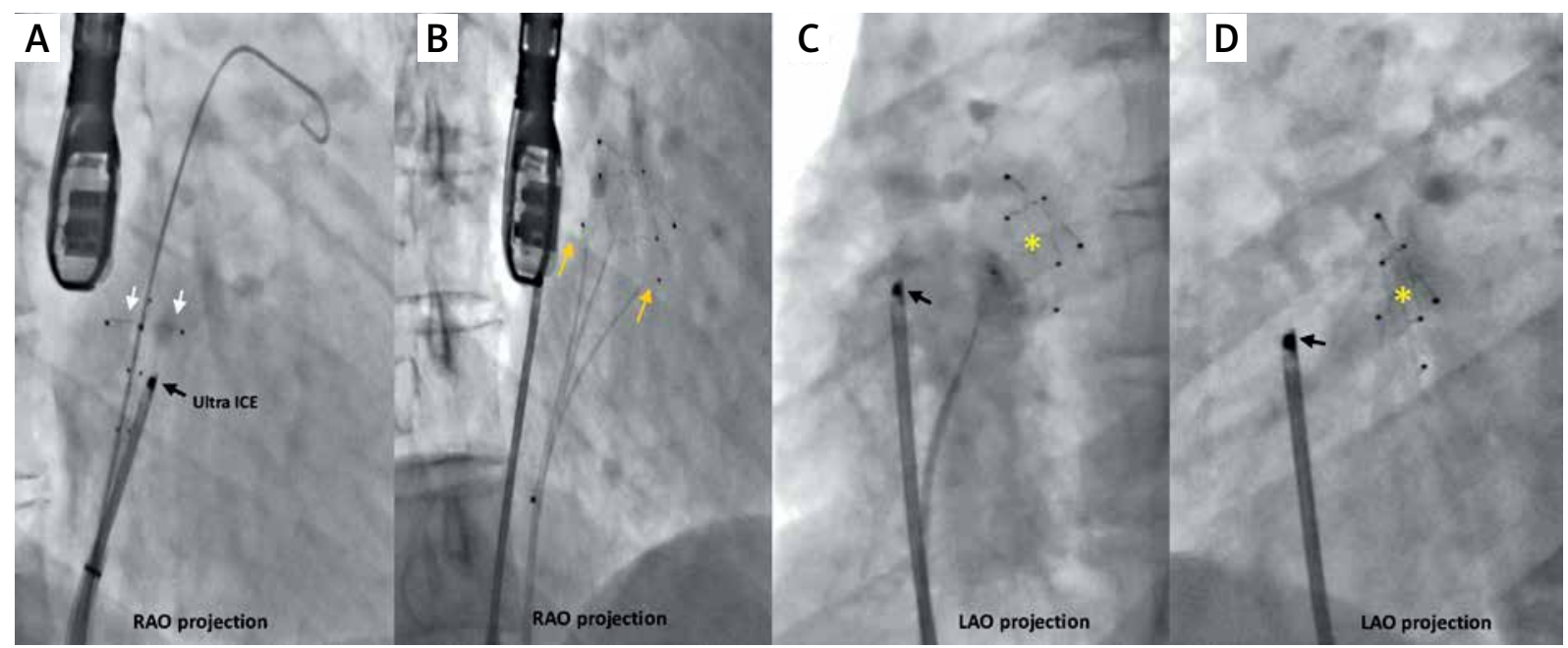

Figure 2. Fluoro-angiographic procedural steps. Over the guide wire placed in the left atrium the Coherex FlatStent EF (FS) PFO closure device, pre-attached to a monorail delivery system by three tethers, is advanced into the left atrium and the distal left anchors (white arrows) are unsheathed and gently retracted to engage the tunnel (A); the body of the device expands within the tunnel, drawing the septum primum and the septum secundum into contact; the proximal right anchors (orange arrows) are opened, engaging on the right side of the tunnel (B); the FS (yellow asterisk) finally deployed (C, D)

Ultra ICE (black arrow) - 9F-9 MHz Ultra ICE catheter-based ultra-sound transducer. 


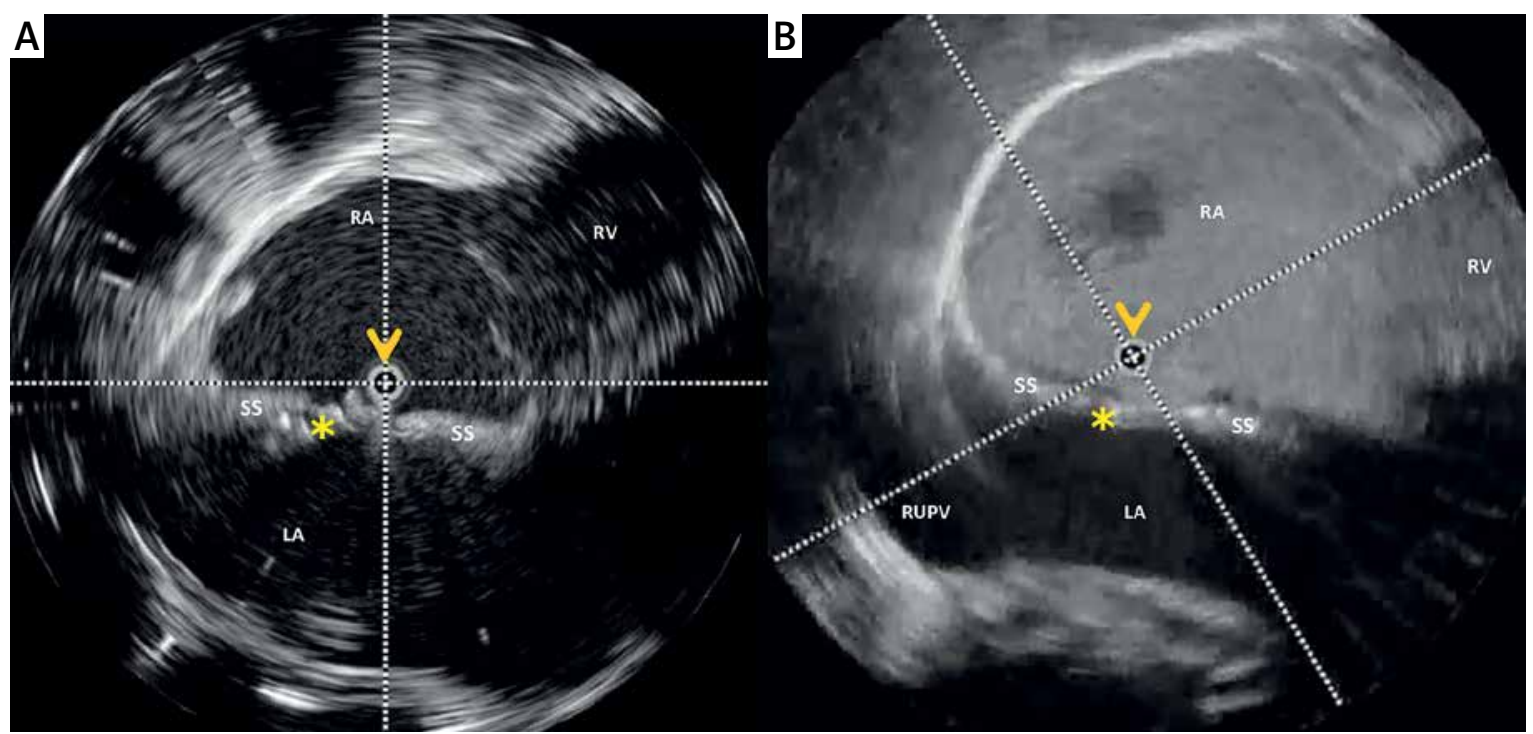

Figure 3. Rotational intracardiac echo by Ultra-ICE (mechanical 9F/9 MHz $360^{\circ}$ scan probe). Intra-procedural views in the long-axis four-chamber plane showing the Coherex FlatStent EF (yellow asterisk) correctly deployed within the PFO tunnel (A) and right-to-left shunt abolition after saline contrast infusion via right femoral vein (B). Ultra ICE catheter (orange arrowhead) is located at the center of the images $R A$ - right atrium, $L A$ - left atrium, $R V$ - right ventricle, RUPV - right upper pulmonary vein, $S S$ - septum secundum.

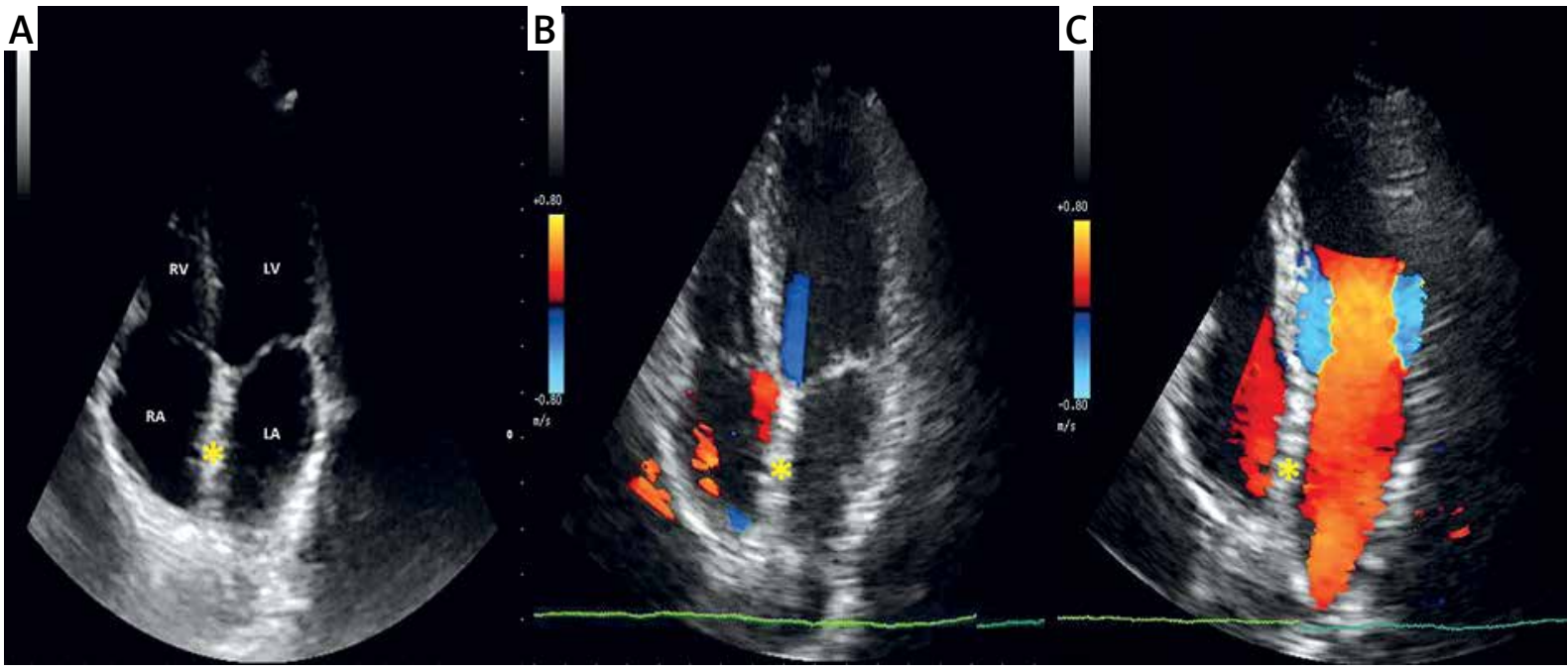

Figure 4. 2D TTE color Doppler follow-up 11 years after the procedure. A, B - apical four-chamber plane showing no residual shunt and the Coherex FlatStent EF (yellow asterisk) embedded into the septum, being impossible to discern the implanted nitinol wire frame covered with polyurethane foam from the native interatrial tissue (C) $R A$ - right atrium, $L A$ - left atrium, $R V$ - right ventricle, $L V$ - left ventricle.

al) will enhance tunnel closure and hopefully increase the in-tunnel closure approach.

\section{Conflict of interest}

The authors declare no conflict of interest.

\section{References}

1. Zanchetta M, Rigatelli G, Pedon L, et al. Intracardiac echocardiography during catheter-based procedures: ultrasound system, examination technique, and image presentation. Echocardiog raphy 2002; 19: 501-7.
2. Ruygrok PN. The Coherex Flatstent: an advance in patent foramen ovale closure. Expert Rev Med Devices 2010; 7: 193-9.

3. Sievert $\mathrm{H}$, Wunderlich N, Reiffenstein I, et al. Initial clinical experience with the Coherex Flatstent and Flatstent EF PFO closure system for in-tunnel PFO closure: results of the Coherex-EU study. Catheter Cardiovasc Interv 2014; 83: 1135-43.

4. Noc M, Cernic Suligoj N, Zvan B, et al. In-tunnel closure of patent foramen ovale with a FlatStent EFTM. Kardiol Pol 2015; 73: 549-56.

5. Aral M, Mullen M. The flatstent versus the conventional umbrella devices in the percutaneous closure of patent foramen ovale. Catheter Cardiovasc Interv 2015; 85: 1058-65. 